\title{
Looking into the crystal ball: From Transistors to the Smart Earth
}

\author{
Alberto Sangiovanni Vincentelli, University of California, Berkeley, USA \\ Donatella Sciuto, Politecnico di Milano, Italy
}

\section{Introduction}

In 2003 at the 40th DAC keynote and then in [ASV2003], the history of Electronic Design Automation was summarized following Giovan Battista Vico, the first philosopher who analyzed history and its patterns. Vico's fundamental contribution was that history repeats itself with a regular pattern. He identified three phases: The age of gods, the age of heroes and the age of men. The age of gods is characterized by knowledge that comes to people from the use of their senses. The age of heroes is characterized by the use of imagination that lets people supersede the sensory information to find the first abstract interpretations of reality. It is the age of creativity, the foundation of great human achievements. The age of men is characterized by reason, during which people fear novelty and creativity as jumps into the dark because no analysis can guarantee any initiative's success.

In 2003 EDA was in the age of men: an age of reason, in which technical innovation slowed down, improvements were incremental and the vendors market became more mature and less risk taking. Nonetheless, contributions EDA made in this age yielded methods and tools to deal with the complexity of designing billions of transistors onto a single chip (starting from the 1 million transistors of the 90's).

The three main vendors, born in the age of heroes, set the stage of EDA market and are still the main players today, and no new major company is competing. The EDA industry had reached maturity.

And today?

\begin{tabular}{|l|c|c|}
\hline & $32 / 28 n m$ node & $22 / 10$ nm node \\
\hline Fab Costs & $\$ 3 \mathrm{~B}$ & $\$ 4 \mathrm{~B}-7 \mathrm{~B}$ \\
\hline Process R\&D Costs & $\$ 1.2 \mathrm{~B}$ & $\$ 2.1 \mathrm{~B}-3 \mathrm{~B}$ \\
\hline Design Costs & $\$ 50 \mathrm{M}-90 \mathrm{M}$ & $\$ 120 \mathrm{M}-200 \mathrm{M}$ \\
\hline Mask Costs & $\$ 2 \mathrm{M}-3 \mathrm{M}$ & $\$ 5 \mathrm{M}-8 \mathrm{M}$ \\
\hline EDA Costs & $\$ 400 \mathrm{M}-500 \mathrm{M}$ & $\$ 1.2 \mathrm{~B}-1.5 \mathrm{~B}$ \\
\hline
\end{tabular}

Table 1 IC Design: Expensive and Difficult (Source IBS)

As shown in Table 1, given the large number of transistors, semiconductor companies are facing a crisis due to the staggering costs of design, which have increasingly being raising in the past few years, in terms of fab costs, process R\&D costs, design costs, mask costs and yes! even EDA costs. These costs make it economically extremely difficult not to say impossible designing small volume parts. Consequently, there have been

- A constant reduction of design starts in favor of standard solutions and of 
customization by software,

- A rationalization of the semiconductor markets whereby system companies, who were important players thirty years ago, exited IC manufacturing and, albeit to a lesser extent, design,

- The fragmentation of the supply chain where IC manufacturing has become the core business of foundries (e.g... TSMC and GlobalFoundries), fabless companies have flourished (e.g., Broadcom and Qualcomm) and IDMs have in part reduced investments in manufacturing by setting up partnership among themselves and with foundries.

To tame this complexity at least in part, a methodology, called IP-based design, has been gaining popularity in the past ten years, whereby a semiconductor is obtained by assembling components from pre-designed parameterized libraries; chips have become printed circuit boards! These libraries include configurable processor cores, memories, special-purpose standard blocks (ASSP), glue logic, and third party special purpose components (Intellectual Property (IP) blocks). IC companies are increasingly adopting this approach that has also enabled new system players, such as Apple, Microsoft, Amazon and Google, to design their own chips. The number of pre-designed IP blocks per chip has increased constantly and has become a flourishing market in itself (see Figure 1)

Digital IP $\quad$ Mixed-signal IP

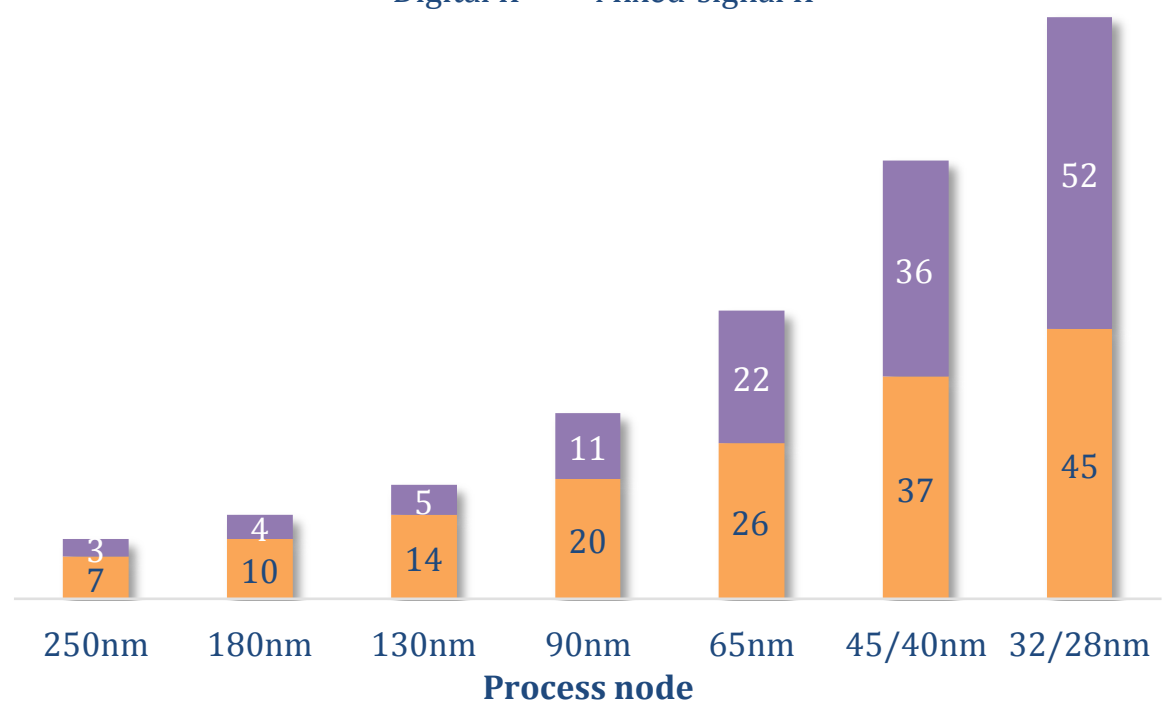

Figure 1 Average number of IP blocks per design (Source Gartner Research)

The IP market has proven to be attractive for EDA vendors because of its tight relationship with design tools as demonstrated in Table 2 where the major IP providers are reported. 


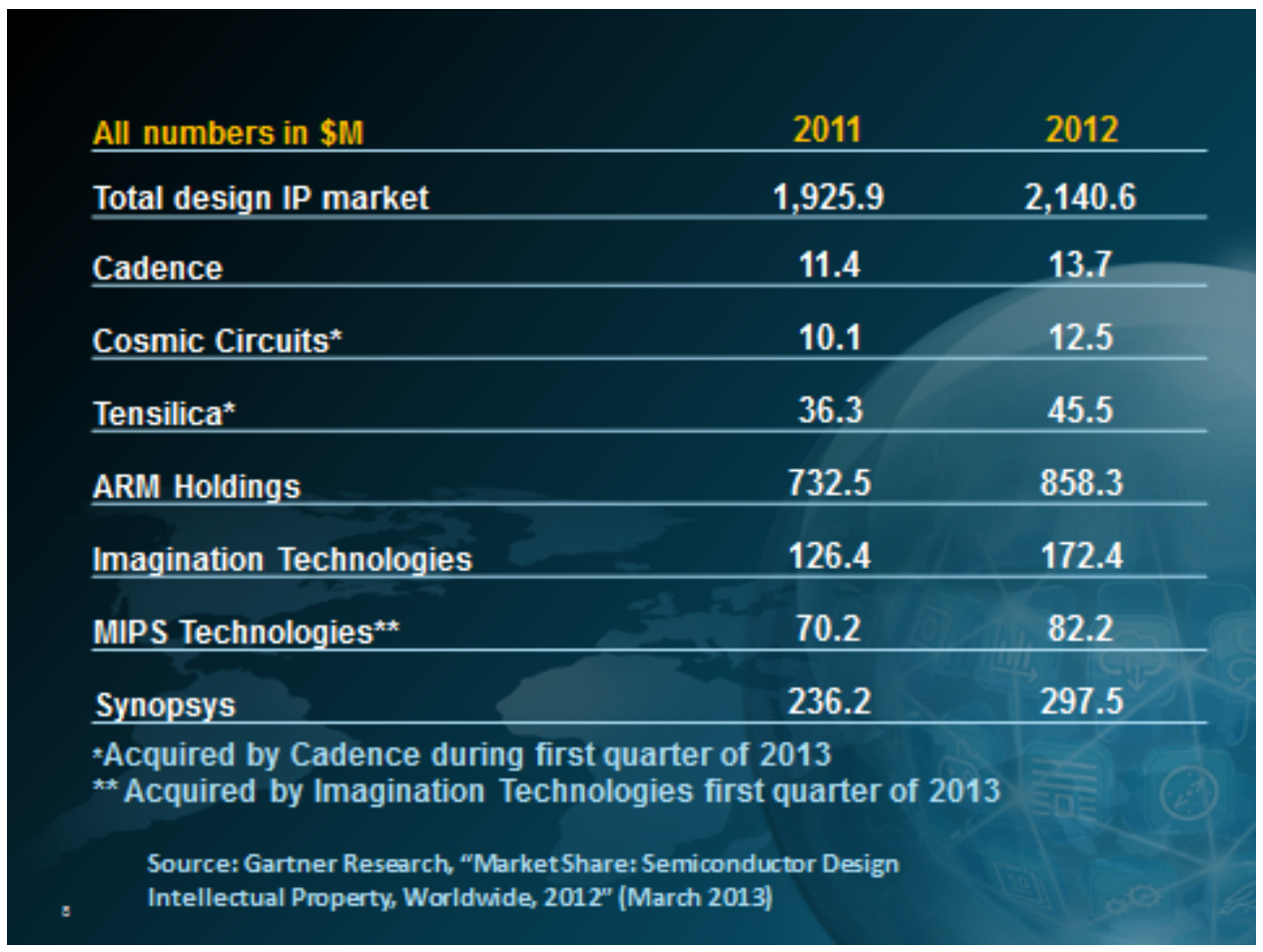

Table 2 IP Market Players

These are some of the major trends in the EDA community but what does our crystal ball shows for the future? Are we going to skip the phase of Gods in Vico's phases and jump directly to the age of Heroes? The next sections are intended to provide some elements to consider when thinking of these questions. 


\section{The Future Environment}

To estimate where EDA will evolve, we need to look at a much bigger picture: what the future of technology and application reserves for us!

\subsection{The Information technology emerging scene}

Information Technology has been rapidly evolving over the years as represented in Figure 2 (due to Jan Rabaey).

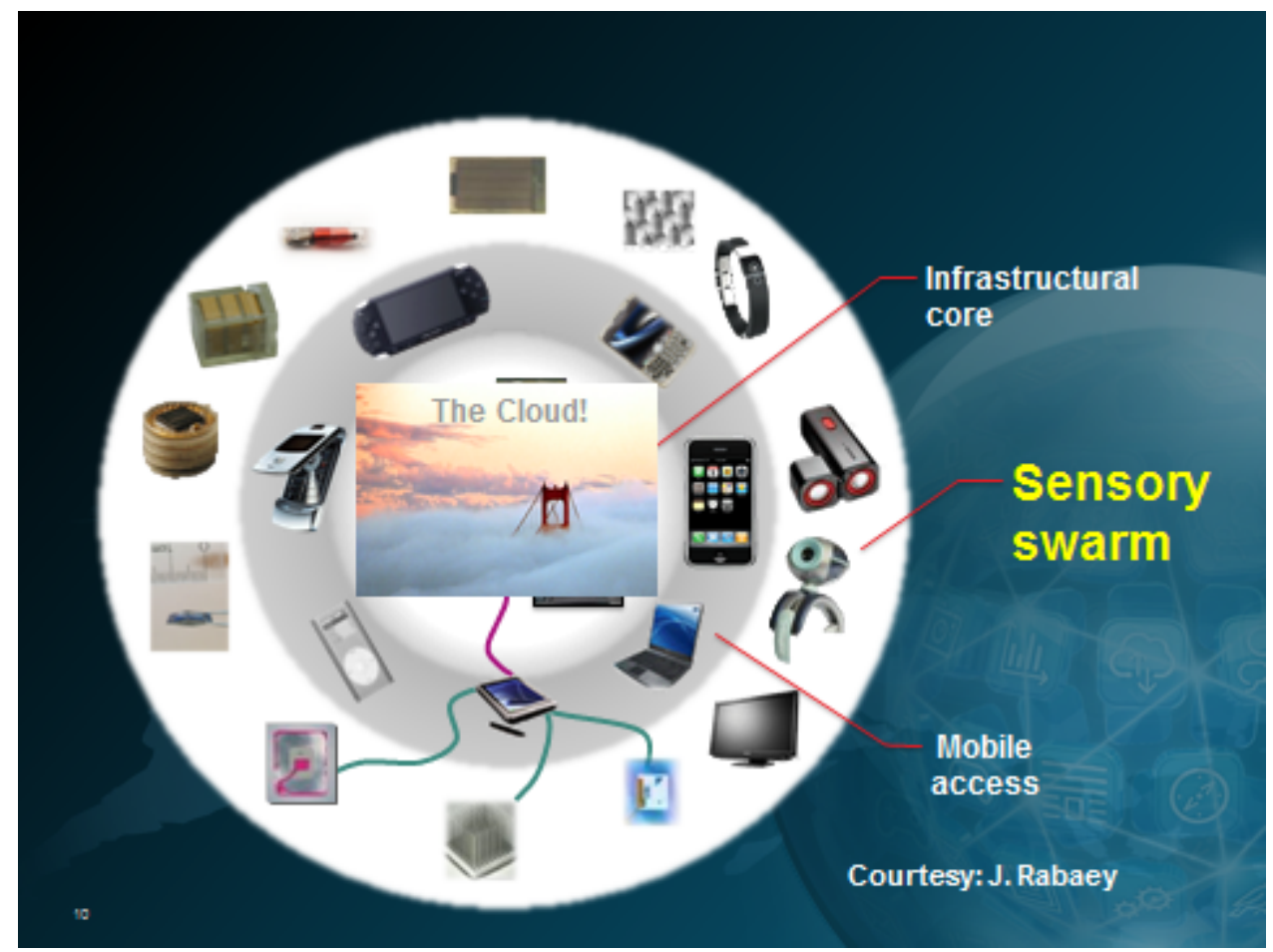

Figure 2. The Emerging IT Scene

In the figure, three layers are identified:

- The center is the infrastructural core that from servers has been moving towards server farms and finally to the cloud where companies such as Amazon, Apple, Google, HP, IBM and Microsoft dominate.

- The intermediate layer consists of mobile smart devices where a fierce battle is played today among companies that either have been the main players for years such as Apple and Samsung, and others such as Google and Microsoft that have recently acquired companies who once were major players such as Motorola and Nokia.

- The outer layer is the one that holds most promise to revolutionize the world as we know it today: the sensory swarm, which will instrument the world enabling a Copernican revolution where the center is once more Man and not the device, where services today unthinkable will be offered to everyone. Market predictions say that by 2025 there will be seven trillion devices serving seven billion people, that is, 1,000 devices per person! This will enable real-life interaction between humans and cyberspace, enabled by enriched input and output devices on and in the body and in the surrounding environment. IBM is already working towards this future with its Smarter Planet Initiative, providing new kind 
of applications. The vision is that the world will be completely instrumented through the sensory swarm, which is interconnected and intelligent. Intelligent systems gather, synthesize and apply information, and can be applied to different areas, as for instance smart water, smart traffic and smart energy.

\subsection{Cyber-Physical Systems (aka Internet of Things, Systems of Systems)}

The evolution of the IT scene has yielded a wide interest in evolving embedded systems in many different directions. Embedded systems can no longer be confined to a single, albeit complex, device such as an engine controller, a braking device or a robotic arm. Cars, airplanes, trains and other transportation systems have become increasingly dependent on a network of embedded systems that needed to communicate to perform tasks such as controlling the operation of the vehicle, monitoring its components for potential faults, and connecting to the external world. This evolution required to think to distributed systems where allocating and coordinating functions to the physical components as well as designing the (wired or wireless) interconnection network became crucial.

Indeed once wireless communication, sensing, computing and control are widely available at low cost, devices could be interconnected together without (or with limited) human interaction. Internet of Things (IoT) (see e.g. [MCK2010]) was born as a concept (albeit, at this time, it is still in its infancy when it comes to actual deployment).

Coordination and collaboration among systems was of particular interest in the defense domain where the agenda for Systems of Systems (SoS) was of importance for battlefield management as well as fleet coordination. Now this concept has been exported to an entire new world of applications including airport management, water distribution systems and the smart grid.

As sensing and control technology grew stronger, the interaction between the physical systems that host the computing and communication components could not be ignored or minimized. In particular, mechanical devices that were actuated with man-activated hydraulic systems (for example, airplanes parts such as ailerons and flaps as well as braking systems and steering) evolved into a totally new architecture where electric motors were controlled automatically by electronic subsystems. The integration of physical systems and processes with networked computing has led to the emergence of a new generation of engineered systems: Cyber-Physical Systems (CPS) (see e.g., [FED2007], [PR02012]). Such systems use computations and communication deeply embedded in and interacting with physical processes such as mechanical, electrical and chemical processes, to add new capabilities to physical systems. These cyber-physical systems range from minuscule (pace makers) to large-scale (the national power-grid). The challenge for CPS is managing dynamics, time, and concurrency in networked, distributed computational and physical systems [DER2012]. With these general characteristics, CPS encompass the features of Internet of Things as well as of Systems of Systems. 


\subsection{The Swarm}

The outer layer of Figure 2 serves as a framework to predict the far future of CPS. A sensory swarm reproduces swarms in nature; in the animal world survival of some species is based on large numbers that provide safety and reliability of the ecosystem. If humans are compared to ants, both of them account for $10-15 \%$ of the terrestrial animal biomass, but while humans have $10^{9}$ neurons per node, ants have $10^{5}$ neurons per node. However ants have been on earth for 1,000 times longer than humans! Large numbers of simple systems imply resiliency and adaptability way superior to smaller numbers of complex systems.

However, as all technologies, sensory swarms have also a downside: such a huge number of devices is easily accessible from potential enemies thus posing open security and privacy issues. In addition, designing applications using the swarm as a platform is a highly complex task because of the sheer number of components, of their diffusion and of the fragility of each component in isolation. Architecting swarm systems, platforms, design methodology and tools are all important tasks in the research agenda to make this vision possible. The Semiconductor Technology Advanced Research Network (STARnet) TerraSwarm Center (http://www.terraswarm.org/) [LEE2014] supported by DARPA and a group of companies (Applied Material, Global Foundries, IBM, Intel, Micron, Raytheon, Texas Instruments and UTC) with headquarters in UC Berkeley is aimed at providing foundational results to overcome these difficulties.

\subsection{Bio-Cyber Systems}

Linking the cyber and the biological worlds is an important research agenda with many implications for the future of mankind and for the health-care and IT industry of tomorrow. Bio-cyber-systems are a combination of biological parts and computing parts. The interaction between humans and devices takes a new dimension: we are moving towards integrated wireless implanted interfaces. For example, devices could be implanted in the brain to provide neurological signals to help people paralyzed to regain mobility, or for replacing brain functionalities lost to diseases, such as Alzheimer and Parkinson, with computing systems. People will be able to control the environment simply by thinking. The implanted interface will then transmit the signals that are needed by the environment to respond to the demand of the users. This approach has the potential of changing radically the way humans and technologies interact.

An experiment along these lines has been already performed in cooperation by MIT and Duke University [LEB2006]. An implanted interface in the brain of the monkey allowed it to control a robotic arm located 600 miles away by connecting the monkey to Internet. More disturbingly, another MIT research [BOY2005] demonstrated that is possible to control the brain with a chip programmed to shine light into specific areas.

\subsection{Synthetic Biology}

Synthetic biology is the science of designing forms of life that do not exist in nature by combining separate elements of DNA to form a coherent system [GIB2004]. Synthetic biology seeks to design biological systems and their components to address a host of problems that cannot be solved using naturallyoccurring entities. The future of biology is then the possibility to combine off- 
the-shelf components to produce new bacteria that can serve different purposes with potential benefits to medicine, environmental remediation and renewable energy. This field is relatively young but has already yielded important results in green energy (a bacterium was created to produce diesel fuel directly from crops instead of ethanol) [PER2012] and medicine (Artemisinin, the active principle of an effective drug to fight malaria, was obtained by bacteria at a fraction of the cost, thus allowing to extend the cure of malaria to very poor populations [R02006]). As in the previous instance, several ethical issues have to be answered, as the potential of engineering life is at the same time exciting and scary.

\subsection{Remarks}

All of the topics briefly outlined above pose immense design problems due to on one side, complexity (billions of devices to connect and use, multi-physics systems requiring the understanding of very heterogeneous mathematical models, unwanted interactions) and ethical issues on the other (safety, security, proper use of the technology, reliability). We can envision that by 2025 integrated components will be approaching molecular limits and every object will be smart. The ensemble will be the function, i.e. the function will not be performed anymore by a single device, but by a collection of devices. The function will be determined by the availability of sensing, actuation, connectivity, computation, storage and energy to make them work. The smaller the devices the less energy is needed and the easier it is to do energy scavenging from the environment. In summary, we will have to deal with humongous networked, distributed, adaptive, hierarchical, hybrid control, computing and communication systems.

Tools that support the design and use of these revolutionary technologies are a necessity. EDA may indeed jump across the Age of the Gods and reach the Age of the Heroes!

\section{The vision for EDA2025}

There is no doubt that the frontier of design automation is in systems, let these be Cyber-Physical Systems, Internet of Things or Systems of Systems. Thus the vision for EDA2025 will be based only on this domain. We do not mean that the challenges posed by integrated circuit technology are serious and that EDA should address them with vigor; we believe these challenges can and will be solved using an evolutionary approach. Not so for the systems we have described in the previous section.

\subsection{System Design in 2013}

Before plunging into the vision for 2025, examine how the system industry is coping today with the problem of designing cyber-physical systems. The state of the art of design is based on the use of a rather old-fashioned water-fall model, the "V" diagram (see Figure 3 The V-DiagramFigure 3). Since the process is sequential, an error found at integration time may have catastrophic effects on schedule and costs. In addition, little formalism, if any, has been used for analysis, let alone synthesis. We believe that in system design, industry at large is at the same stage of the IC industry 20-30 years ago. 


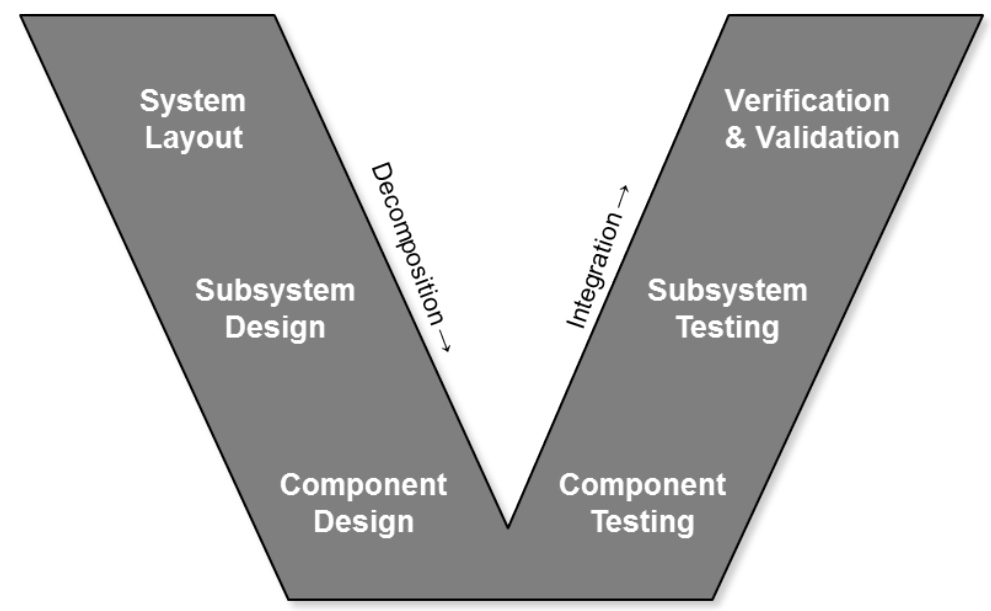

Figure 3 The V-Diagram

EDA has dealt with very complex integrated circuits and is based on the fundamentals of design: formalization, modeling, simulation, formal analysis, layers of abstraction, verification and synthesis. EDA is in a sense rooted on the science of design. As such, some principles should be abstracted and transported in system design.

\subsection{The Principles of EDA}

The EDA fundamental principles are few and powerful [ASV2003]: abstraction (e.g., from transistors to logic level) and tools that derive from these abstractions and provide effective ways to master complexity. However, the real fundamental breakthrough in the VLSI era was a rigorous methodology, where for us methodology is a way to limit the choice of designers in the solution space: a methodology in some sense is freedom from choice.

The general principles for dealing with complexity in EDA have been:

- Decomposition: reduce the number of items to consider by breaking the design object into semi-independent parts (divide et impera);

- Composition: obtain a solution from available parts;

- Abstraction: reduce the number of items by aggregating objects and by eliminating unnecessary details with respect to the goal at hand.

- Refinement: proceed from an abstract representation of the design towards an implementation by adding details.

Complexity has been also managed by "construction": by constraining "artificially" the design space (i.e., imposing regular layout, or synchronous designs), and starting high in the abstraction layers, it is possible to define a number of refinement steps that go from the initial description to the final implementation that can be performed automatically with the use of sophisticated tools. For example, the digital design flow in use today marches from a high-level description of the function to be performed using appropriate languages towards a synchronous gate-level representation obtained by logic synthesis to a transistor layout, via automatic place and route made possible by restricting the position of transistors in regular patterns that are stored in appropriate, fully characterized libraries. 


\subsection{Platform-Based Design as a Paradigm for System Design}

Platform-based design has been advocated since the end of the 80's for electronic design, and used today for cyber-physical systems. In this case the library of components is not gates but multi-physics components [ASV2007].

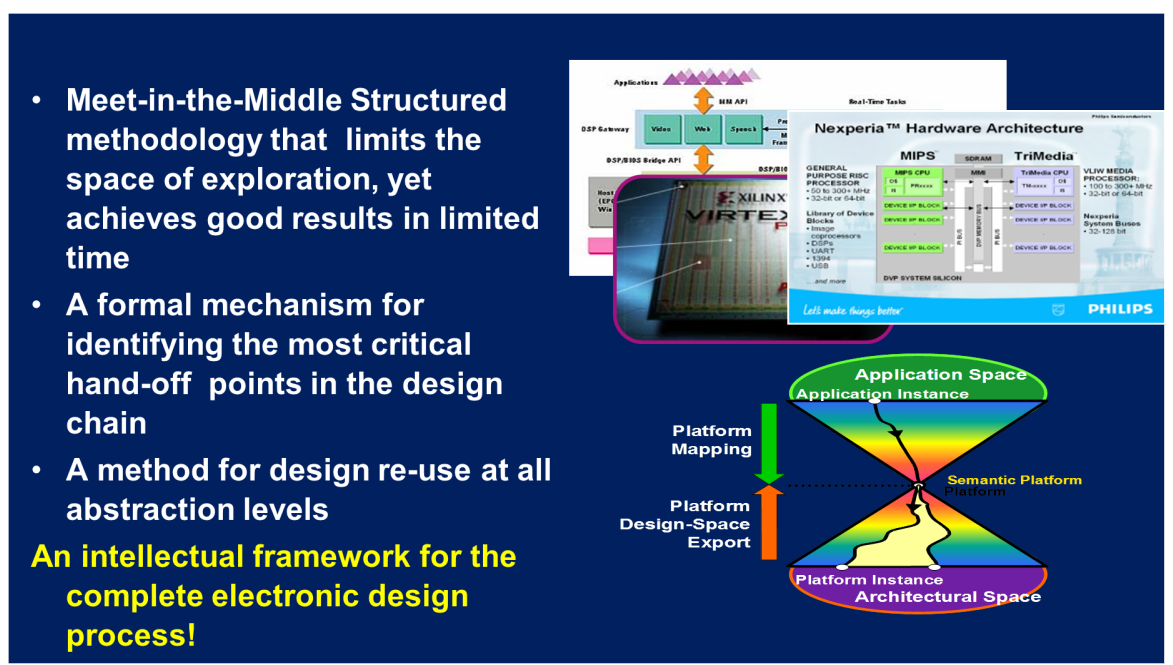

Figure 4 Platform-Based Design

The principles are the same: the concept of platform as an "opaque" layer of abstraction that exposes the critical parameters of the layers below to the layer above is an essential part of a design methodology for supporting the design of systems composed of parts, such that when you integrate them you achieve a system that works. Platform-based design is a sequence of steps taken from the requirements formalization to its realization, in a meet-in-the-middle approach. In the bottom-up phase you have to choose the components that allow satisfaction of the requirements defined in the top-down phase. In fact, when we have a common semantic domain that can be used to represent both the top and the bottom layer of abstraction in the stack, then we can always formulate the selection of the best lower-level platform as a covering problem, similarly as what is done in logic synthesis. When this methodology is applied to complex cyber-physical system it is necessary to make sure that your design is correct. There is the need to explicit the conditions and assumptions on the composition of the different components [ASV2012]. Contracts are assume-guarantee pairs that explicit that component properties are guaranteed under a set of assumptions on its environment. From these, global properties of systems are derived based on local properties of the components. Contracts represent a key notion for formalizing and analyzing system requirements.

The principles of platform-based design are general principles and can be applied in designing avionic systems (such as complex electrical power systems in airplanes) or in designing intelligent buildings to achieve, for instance, energy efficiency.

Platform-based design has a role also in the Swarm. Indeed, TerraSwarm [LEE2014] views the swarm as a set of resources composed of all sorts of computing, sensing and actuating devices that can be used by a set of applications that could be for example, home security, energy efficiency management, and health monitoring. The point is how to couple applications and 
resources. To do so we need a "mediation" layer called the swarm operating system that present a uniform "API" to the applications. The mediation layer can be considered the "system abstraction" of platform-based design and the design methodology for the swarm is then considered as an instance of PBD.

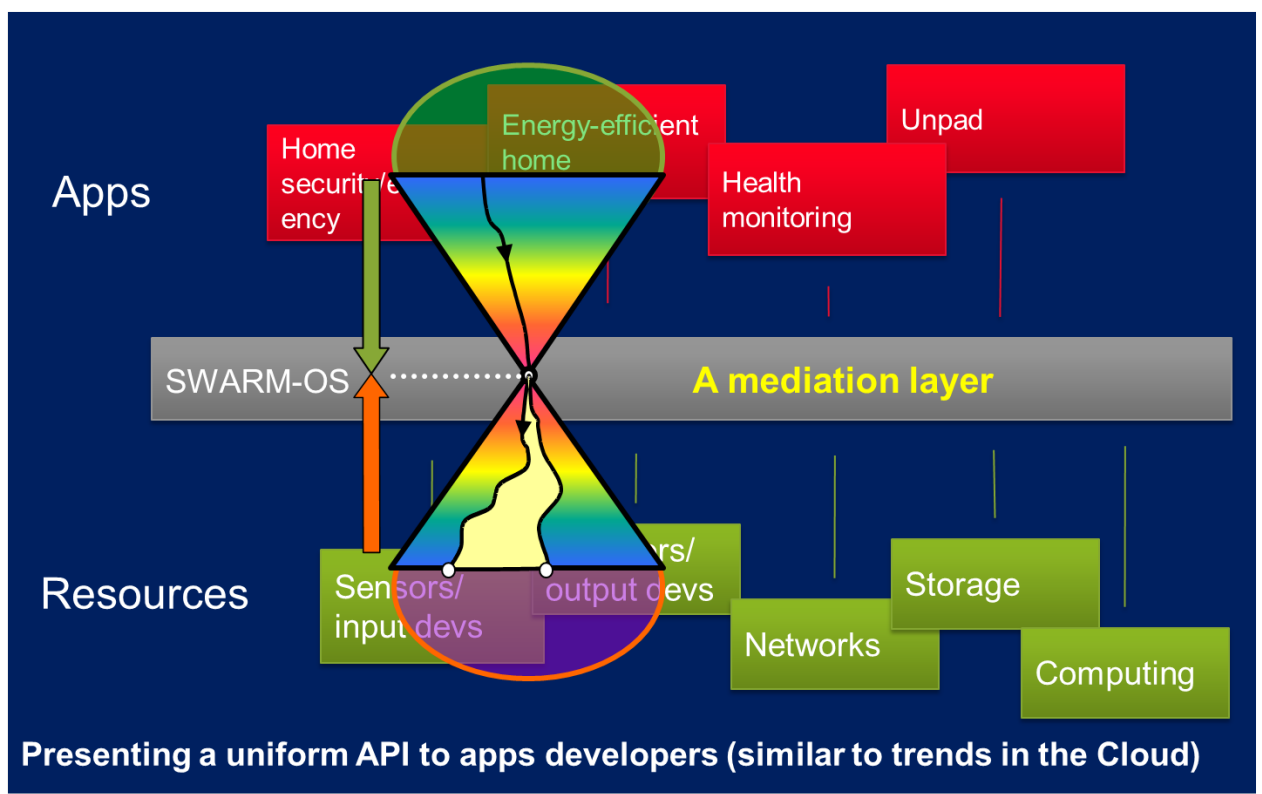

Figure 5 The Swarm as a Platform (courtesy of Jan Rabaey)

Similarly, the principles of platform-based design can be used for designing synthetic biological systems, in which standardized biological parts represent the components that can be used to create biological systems based on a set of requirements [DEN2012]. Clotho is an example of such a design environment, and it is composed of four main steps:

- Decide on the general functionality desired by the biological systems

- Specify the composition of the elements and the constraints on the system by identifying contracts;

- Design variations of the design, assign theoretical parts to physical samples, modify sequences, etc., i.e. mapping of the functionalities onto your set of objects library (DNA segments),

- Send the design to liquid handling robot assembly workflows, capture successes and failures as constraints for future designs, and save created devices.

These steps are very similar to the IC design flow. The methodology is exactly the same. This is the knowledge of EDA that can be put to work in different application domains. 


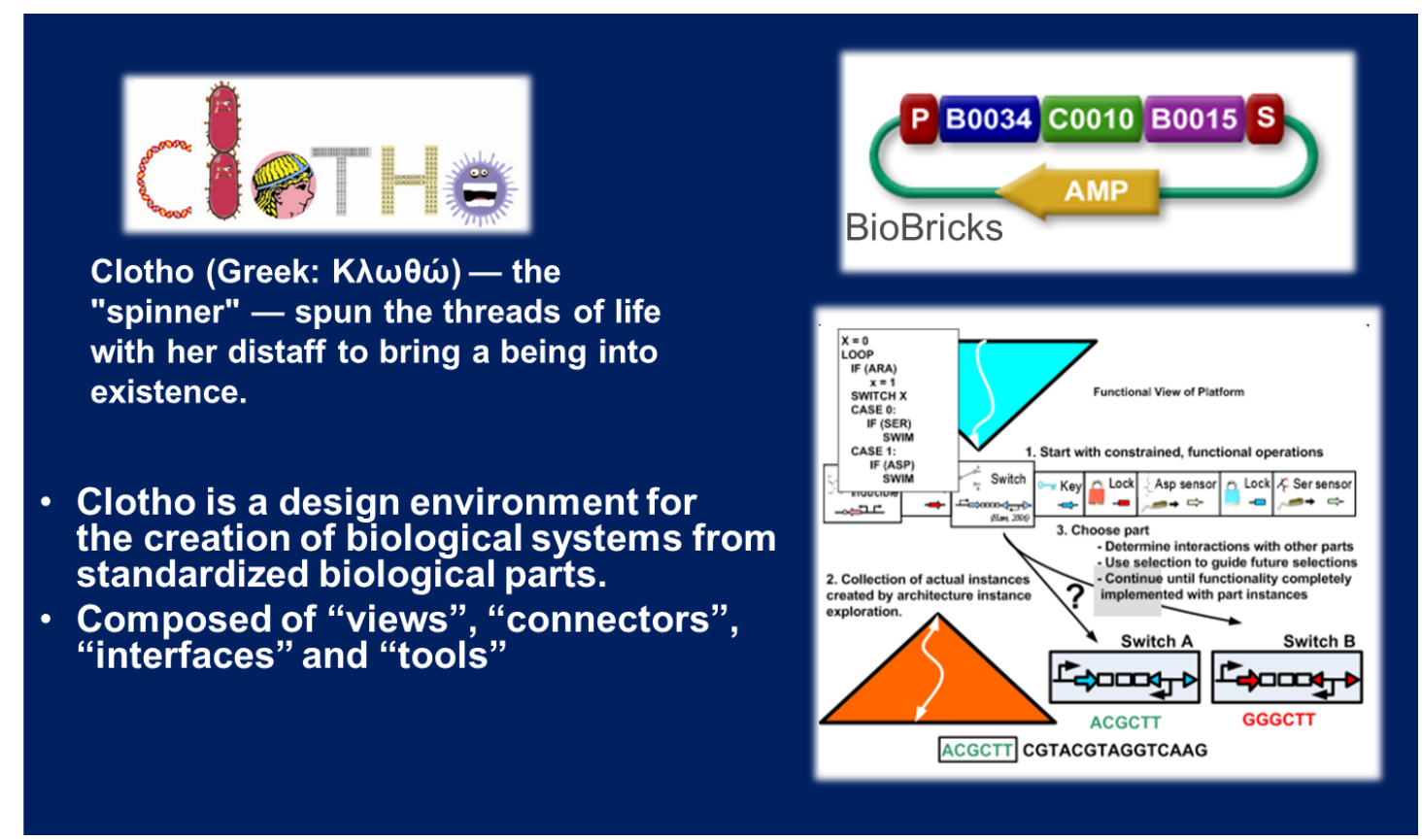

Figure 6 PBD environment for synthetic biological systems (courtesy of Doug Densmore)

\section{Conclusions}

EDA is a unique wonderful field where search for the essential, algorithms, innovation and business come together. Can EDA and embedded systems expand into new fields? We need new paradigms to go beyond what we know to solve the problems of designing cyber-physical systems, systems of systems up to the scale of the swarm systems. To do that we need to support the design chain and we need to help industry to decide if they need to be vertically integrated or horizontally integrated. We need to identify methodologies to build brain-machine interfaces to be able to control the environment directly with our thoughts through the sensory swarm. And finally synthetic biology is opening a new vista on how to master and use nature. These are the opportunities for EDA.

We would like to end with another quote by Vico, which we believe can be a source of inspiration to us all. He characterized the age of heroes as "The holy furor for truth that lives in the eternal attempt to go beyond the limit, in the infinite possibility of self-realization and of overtaking ourselves to discover the power of the spirit and give a new push towards knowledge." Let us make an effort to live up to these words.

\section{References}

[ASV2003] A. Sangiovanni Vincentelli, "The Tides of EDA", IEEE Design \& Test of Computers, pp. 59-75, November-December 2003, vol.20, issue 6.

[FED2007] Federal Networking and Information Technology - R\&D Program. Leadership Under Challenge: Information Technology $R \& D$ in $a$ 
CompetitiveWorld. Technical report, Council of Advisors on Science and Technology, 2007.

[PROC2012] Special Issue on Cyber - Physical Systems, Proceedings of the IEEE, Vol. 100, n. 1, Jan. 2012.

[MCK2010] Michael Chui, Markus Löffler, and Roger Roberts, The Internet of Things, McKinsey Quarterly, March 2010.

[DER2012]P. Derler, E. Lee, and A. Sangiovanni Vincentelli, Modeling cyber physical systems, Special Issue on Cyber - Physical Systems, Proceedings of the IEEE, Vol. 100, n. 1, Jan. 2012, pp.13-28.

[DW02006] Cynthia Dwork, Frank McSherry, Kobbi Nissim, and Adam Smith. Calibrating noise to sensitivity in private data analysis. In Shai Halevi and Tal Rabin, editors, Theory of Cryptography, volume 3876 of Lecture Notes in Computer Science, pages 265-284, Springer Berlin / Heidelberg, 2006.

[BOY2005] Boyden, E. S.; Zhang, F.; Bamberg, E.; Nagel, G.; Deisseroth, K. Millisecond-timescale, genetically targeted optical control of neural activity, Nat. Neuroscience 8 (9): 1263-8.

[GIB2004] W. Wayte Gibbs Synthetic Life, Scientific American, May 2004.

[PER2012] P. P. Peralta-Yahya, F. Zhang, S. B. del Cardayre, and J. D. Keasling, Microbial engineering for the production of advanced biofuels, Nature 488:320$328,2012$.

[R02006] D-K. Ro, E. M. Paradise, M. Ouellet, K. J. Fisher, K. L. Newman, J. M. Ndungu, K. A. Ho, R. A. Eachus, R. S. Ham, J. Kirby, M. C. Y. Chang, S. T. Withers, Y. Shiba, R. Sarpong, and J. D. Keasling, "Production of the antimalarial drug precursor artemisinic acid in engineered yeast, Nature 440:940-943, 2006

[ASV2007] Alberto Sangiovanni-Vincentelli. Quo vadis SLD: Reasoning about trends and challenges of system-level design. Proceedings of the IEEE, 3:467-506, March 2007.

[ASV2012] Alberto Sangiovanni-Vincentelli, Werner Damm, and Roberto Passerone. Taming Dr. Frankenstein: Contract-Based Design for Cyber-Physical Systems, European Journal of Control, 18(3):217\{238, June 2012.

[LEE2014] Edward A. Lee, Jan Rabaey, David Blaauw, Prabal Dutta, Kevin Fu, Carlos Guestrin, Bj orn Hartmann, Roozbeh Jafari, Doug Jones, John Kubiatowicz, Vijay Kumar, Rahul Mangharam, Richard M. Murray, George Pappas, Kris Pister, Anthony Rowe, Alberto Sangiovanni-Vincentelli, Sanjit A. Seshia,Tajana Simunic Rosing, Ben Taskar, John Wawrzynek, David Wessel, The Swarm at the Edge of the Cloud, IEEE Design and Test, to appear, 2014

[DEN2014] D. Densmore and S. Hassoun, Design Automation for Synthetic Biological Systems, IEEE Design \& Test of Computers, vol. 29, iss. 3, pp. 7-20, 2012 